\title{
Effects of Dietary Cholesterol and Fat Saturation on Plasma Lipoproteins in an Ethnically Diverse Population of Healthy Young Men
}

\author{
Christopher J. Fielding, ${ }^{*}$ Richard J. Havel, ${ }^{\ddagger}$ Karen M. Todd, ${ }^{\S}$ Kyee E. Yeo, Monique C. Schloetter, ${ }^{\S}$ Vivian Weinberg, $^{\S}$ \\ and Philip H. Frost" \\ Cardiovascular Research Institute and Departments of $*$ Physiology, ${ }^{\ddagger}$ Medicine, and ${ }^{8}$ General Clinical Research Center, University of \\ California Medical Center, San Francisco, California 94143
}

\begin{abstract}
The individual effects of dietary cholesterol and fat saturation on plasma lipoprotein concentrations were determined in an ethnically diverse population of normolipidemic young men ( 52 Caucasian, 32 non-Caucasian). The experimental diets contained $\sim 200$ or $600 \mathrm{mg} / \mathrm{d}$ of cholesterol, 36-38\% of calories as fat, and high or low proportions of saturated and polyunsaturated fat (polyunsaturated/saturated fat ratio $\sim 0.8$ vs 0.3 ). At the lower cholesterol intake, the high saturated fat diet had only a modest effect on LDL cholesterol in Caucasians $\left(+6 \mathrm{mg} / \mathrm{dl}^{-1}\right)$ and none in non-Caucasians. $600 \mathrm{mg}$ cholesterol with high saturated fat led to a substantial mean increase in LDL cholesterol, which was significantly greater in Caucasian than in non-Caucasian subjects (+ $31 \mathrm{mg} / \mathrm{dl}$ vs $16 \mathrm{mg} / \mathrm{dl}, P<0.005) .600 \mathrm{mg}$ cholesterol with increased polyunsaturated fat gave a mean LDL increase of $16 \mathrm{mg} / \mathrm{dl}$, lower than found when the same high cholesterol intake was coupled with increased saturated fat. Variation in cholesterol rather than the proportions of saturated and polyunsaturated fat had the most influence on LDL-cholesterol levels. Among non-Caucasians it was the only significant factor. (J. Clin. Invest. 1995. 95:611618.) Key words: cholesterol - dietary fats - lipoproteins • LDL-cholesterol • cholesterol acyltransferase
\end{abstract}

\section{Introduction}

Changes in dietary fat saturation and cholesterol content, alone or in combination, have often been demonstrated to modify plasma cholesterol and, in particular, that in $\operatorname{LDL}(1,2)$. That is, transfer to a diet rich in cholesterol and saturated fat usually increases LDL cholesterol (LDL-C) ${ }^{1}$ while diets reduced in

Address correspondence to C. J. Fielding, L-1341, Cardiovascular Research Institute, Box 0130, University of California Medical Center, San Francisco, CA 94143. Phone: 415-476-4307; FAX: 415-476-2283.

Received for publication 20 August 1992 and in revised form 30 October 1994.

1. Abbreviations used in this paper:CETP, cholesteryl ester transfer protein; EC, esterified cholesterol; FC, free cholesterol; HC-PUFA, high cholesterol polyunsaturated fat; HC-SFA, HC saturated fat; IDL, intermediate density lipoprotein; LCAT, lecithin: cholesterol acyltransferase; LC-PUFA, low cholesterol PUFA; LC-SFA, low cholesterol SFA; LDLC, LDL cholesterol; P/S, polyunsaturated/saturated fat ratio; TC, total cholesterol; TG, triglyceride.

J. Clin. Invest.

(C) The American Society for Clinical Investigation, Inc.

0021-9738/95/02/0611/08 \$2.00

Volume 95, February 1995, 611-618 cholesterol and enriched in unsaturated fat have an opposite effect. Some investigators have concluded that the effect of saturated fat is most important (3-5). Others have concluded that dietary cholesterol may be more important, particularly when the diet is rich in saturated fat (6-8). Additionally, individual saturated fatty acids can have variable effects on LDLC $(9,10)$.

The origin of cholesterol in LDL has recently become much better defined. Much of the cholesteryl ester in plasma is synthesized in HDL from lipoprotein or cell membrane free cholesterol by the action of lecithin:cholesterol acyltransferase (LCAT) and part of this is transferred, by the action of cholesteryl ester transfer protein (CETP) to VLDL or its products, intermediate density lipoprotein (IDL) and LDL (11). However newly secreted plasma VLDL, the precursor of plasma LDL, already contains significant amounts of cholesteryl ester (12). The activities of both LCAT and CETP are increased postprandially $(13,14)$. LCAT and CETP activities are also modified by diet (15). The cholesterol content of newly secreted VLDL may also be under dietary control. Changes in plasma LDL-C concentrations could be the result of changes in the activities of LCAT or CETP in plasma, the cholesterol content of newly secreted VLDL, or the rate of removal of LDL from the circulation (16-18).

In the study reported here, we asked whether the amount of cholesterol consumed, or the saturation of dietary fat, was more important in determining plasma and LDL cholesterol level; and whether changes in plasma cholesterol metabolism in fasting and postprandial plasma could explain changes in plasma cholesterol concentration.

Most studies of the effects of dietary lipids on plasma lipoproteins have been carried out in populations either largely Caucasian or undefined. Because our study population was ethnically diverse, we could evaluate the influence of ethnicity on the response to dietary fat and cholesterol. The data we obtained suggest that it may have a significant role, particularly in response to saturated fat.

\section{Methods}

Selection criteria. Individuals recruited to this study were healthy, nonsmoking males (25-35 yr). We excluded those with plasma cholesterol levels above the 90th percentile or below the 5th percentile for age, those with triglycerides above the 80th percentile (19), or those with a history of systemic disease such as diabetes or renal failure. Men whose body weight deviated more than $10 \%$ from their ideal body weight range for height and frame size (based on the 1983 Metropolitan Life Insurance tables) were also excluded. The protocol, all procedures, and consent forms were reviewed and approved by the University of California Committee on Human Research.

Food Intake. Subjects consumed only meals prepared individually in the General Clinical Research Center. Three meals per day plus a 
Table I. Chemical Analysis of Nutrients for the Four Lipid Study Diets

\begin{tabular}{|c|c|c|c|c|}
\hline Nutrient & LC-PUFA & LC-SFA & HC-PUFA & HC-SFA \\
\hline kcal & $3,018 \pm 109$ & $3,144 \pm 61$ & $3,088 \pm 146$ & $3,049 \pm 50$ \\
\hline Protein (g) & $113.3 \pm 14.2$ & $99.2 \pm 1.8$ & $116.7 \pm 12.1$ & $106.5 \pm 12.7$ \\
\hline Fat (g) & $123.1 \pm 3.0$ & $132.6 \pm 2.5$ & $132.2 \pm 8.3$ & $120.3 \pm 9.9$ \\
\hline Carbohydrate (g) & $363.5 \pm 32.9$ & $388.1 \pm 17.5$ & $357.8 \pm 6.1$ & $384.1 \pm 21.7$ \\
\hline Saturated Fat (g) & $35.8 \pm 2.7$ & $55.5 \pm 1.4$ & $43.6 \pm 3.8$ & $58.9 \pm 6.4$ \\
\hline \multicolumn{5}{|l|}{ Polyunsaturated } \\
\hline Fat (g) & $29.7 \pm 1.2$ & $21.1 \pm 3.6$ & $35.2 \pm 3.5$ & $16.4 \pm 0.9$ \\
\hline \multicolumn{5}{|l|}{ Monounsaturated } \\
\hline Fat (g) & $57.6 \pm 2.8$ & $56.0 \pm 4.4$ & $53.4 \pm 3.8$ & $44.8 \pm 6.2$ \\
\hline Cholesterol (mg) & $175.5 \pm 40.2$ & $202.5 \pm 3.3$ & $602.8 \pm 55.8$ & $635.4 \pm 45.1$ \\
\hline \multicolumn{5}{|l|}{ Percentage kcal } \\
\hline Protein & $15.1 \pm 2.2$ & $12.6 \pm 0.2$ & $15.1 \pm 0.90$ & $14.0 \pm 1.9$ \\
\hline Percentage kcal & & & & \\
\hline Carbohydrate & $48.1 \pm 3.0$ & $49.4 \pm 1.4$ & $46.4 \pm 1.4$ & $50.4 \pm 2.2$ \\
\hline \multicolumn{5}{|l|}{ Percentage kcal } \\
\hline Fat & $36.7 \pm 0.7$ & $38.0 \pm 1.1$ & $38.5 \pm 1.0$ & $35.5 \pm 2.9$ \\
\hline $14: 0(\mathrm{~g})$ & $2.7 \pm 0.5$ & $5.3 \pm 0.6$ & $2.9 \pm 0.8$ & $7.6 \pm 1.3$ \\
\hline $16: 0(\mathrm{~g})$ & $20.3 \pm 1.8$ & $27.9 \pm 1.2$ & $24.2 \pm 2.3$ & $30.9 \pm 3.0$ \\
\hline $18: 0(\mathrm{~g})$ & $9.6 \pm 0.9$ & $16.0 \pm 0.8$ & $12.7 \pm 0.2$ & $14.1 \pm 1.1$ \\
\hline $16: 1(\mathrm{~g})$ & $1.2 \pm 0.3$ & $1.8 \pm 0.4$ & $1.4 \pm 0.5$ & $2.0 \pm 0.1$ \\
\hline $18: 1(\mathrm{~g})$ & $55.5 \pm 2.5$ & $52.7 \pm 4.3$ & $51.4 \pm 3.5$ & $41.4 \pm 6.0$ \\
\hline $18: 2(\mathrm{~g})$ & $27.5 \pm 1.0$ & $19.0 \pm 2.8$ & $32.0 \pm 3.4$ & $15.0 \pm 1.3$ \\
\hline $18: 3(\mathrm{~g})$ & $2.2 \pm 0.5$ & $2.1 \pm 0.8$ & $3.0 \pm 0.1$ & $1.4 \pm 0.1$ \\
\hline
\end{tabular}

Total fat calculated from composites. Saturated, monounsaturated and polyunsaturated fat represent the total mass of fatty acids. In the report of individual fatty acids, gas-liquid chromatography peaks containing $<0.2 \%$ of total fatty acids are not included. Values are means $\pm S D$ of the 3-d diet cycle. Diet composites were analyzed by Hazelton Labs America, Madison, WI.

snack were provided. The caloric content of the food was calculated to maintain body weight. Body weight was maintained within 3 lbs of starting weight for each individual throughout the 6-wk study. Energy requirements were estimated from the Harris-Benedict equation (20) and a detailed physical activity questionnaire. Body weights were measured daily, and exercise levels were kept constant and monitored by the dietician. All food was prepared using recipes designed for this study; no formulas or liquid supplements were used.

Experimental design. Upon first entry into the study, all subjects consumed for a 2-wk washout period a baseline diet designed to contain $\sim 200 \mathrm{mg} / \mathrm{d}$ cholesterol and a high proportion of unsaturated fat. The compositions of this diet (low cholesterol, high polyunsaturated fat diet [LC-PUFA]) and others consumed in this study were confirmed by chemical analysis (Table I). Subjects were consecutively assigned to one of four diet groups after the washout period. One group continued to eat the baseline (LC-PUFA) diet for a further $4 \mathrm{wk}$ (Fig. 1). The other three groups changed at that time to diets low in cholesterol but high in saturated fat (LC-SFA), high in cholesterol and high in saturated fat (HC-SFA), or high in cholesterol and high in polyunsaturated fat (HC-PUFA) (Table I).

At the beginning (day 1 ) and the end (day 15) of the 2-wk washout period, blood was collected after an overnight fast. Postprandial blood sampling was done after each subject consumed a test meal. For this and all subsequent postprandial tests (Fig. 1) the test meal consisted of one third of daily calories, fat, and cholesterol of the diet being consumed during the previous two wk. On days 1 and 15 an LC-PUFA test meal was consumed. Further blood samples were drawn 3, 6, 9, and $12 \mathrm{~h}$ postprandially. During this period no food was taken. In each case, blood was withdrawn into ice-cooled plastic tubes containing sodium citrate ( $\mathrm{pH} 7.0$, final concentration $0.01 \mathrm{M}$ ) and immediately cooled at $0^{\circ} \mathrm{C}$ to inhibit esterification of free cholesterol by LCAT or the transfer of preformed cholesteryl esters among plasma lipoprotein classes. Plasma was obtained by centrifugation at $2-4^{\circ} \mathrm{C}(2,000 \mathrm{~g}, 30$
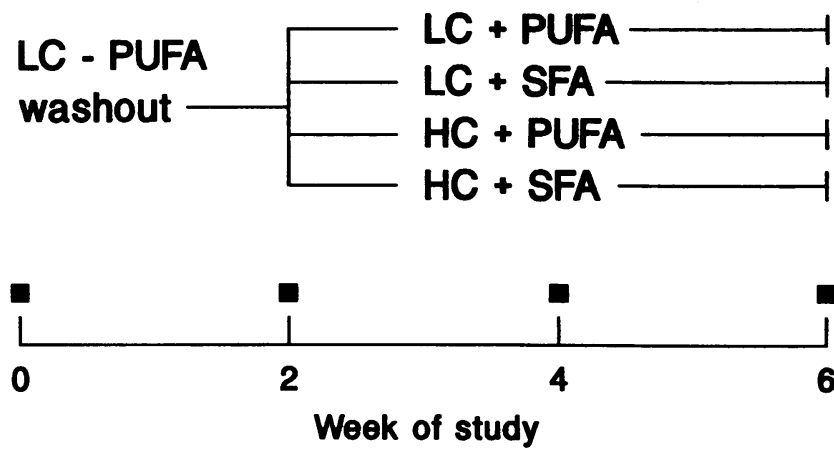

Figure 1. Experimental design. Squares indicate the timing of fasting and postprandial blood sampling. Diets consumed in the washout and experimental periods are described in Table $I$.

min) and used in the assays described below. In addition, at weeks 4 and 6 of the study, i.e., after 2 and then 4 wk of the experimental diets, fasting plus four postprandial blood samples at 3-h intervals were again obtained after a test meal.

Determination of plasma lipoprotein concentrations. Plasma and lipoprotein total and free cholesterol (TC, FC) and triglyceride (TG) were determined by automated enzymatic assays with an autoanalyzer (Cobas Mira; Roche Laboratories, Nutley NJ) $(21,22)$; except that in the case of VLDL cholesterol levels, where the high postprandial concentration of triglyceride-rich particles affected the linearity of the enzymatic cholesterol assay, an ABA-100 chromatic analyzer (Abbott Laboratories, North Chicago, IL) was used. All assays were routinely calibrated against plasma samples provided by the Center for Disease Control, Atlanta, GA. Total cholesterol and triglyceride were measured with reagents supplied by Roche Diagnostics (Branchburg, NJ); free cholesterol was determined with cholesterol oxidase (Wako Chemicals, Richmond, VA). Plasma free and total HDL cholesterol were assayed in plasma after precipitation of VLDL and LDL with dextran sulfate and $\mathrm{Mg}^{++}$(23). Portions of plasma were centrifuged after adjustment of density to 1.006 or $1.019 \mathrm{~g} / \mathrm{ml}$. VLDL total cholesterol and triglyceride were determined by autoanalyzer on the $d 1.006 \mathrm{~g} / \mathrm{ml}$ supernatant fraction (24). IDL $(1.006<d<1.019 \mathrm{~g} / \mathrm{ml})$ was determined as the difference between the concentration of cholesterol or triglyceride in the $d 1.019$ and $d 1.006 \mathrm{~g} / \mathrm{ml}$ supernatant fractions. LDL lipid was determined as: (plasma - [HDL + IDL + VLDL]). Triplicate samples were assayed in each case, with a coefficient of variation of $<2 \%$. Plasma and lipoprotein lipid values are expressed as milligrams per deciliter plasma.

Determination of LCAT and CETP activities. LCAT activity was determined as the rate of decrease of plasma FC mass with time at $37^{\circ} \mathrm{C}$ in the presence of $10 \mathrm{mM}$ Tris-HCL buffer, $1 \mathrm{mM}$ disodium EDTA (pH 7.4) (25). This is equivalent to the rate of increase of total plasma esterified cholesterol (EC). In the same plasma samples we also measured the rate at which $\mathrm{EC}$ increased in $\mathrm{HDL}$ at $37^{\circ} \mathrm{C}$. The rate of EC transfer to VLDL and LDL from HDL (26) was then calculated as:

[Increase in VLDL, LDL EC] = [Increase in total plasma EC - increase in HDL EC].

Pentuplicate samples were assayed at each time point, with a coefficient of variation of $<1.0 \%$ in each case.

Data analysis. The design of this study required a 2-wk washout phase followed by $4 \mathrm{wk}$ of an experimental diet. To avoid possible variability due to the habitual diet all subjects received the identical LC-PUFA washout diet. The purpose of this design was to attempt to achieve a common baseline for all subjects.

The autoanalyzer output was captured into computer (IBM80; IBM Corp., Armonk, NY) using custom software. The statistical analyses were performed using the SAS statistical software package (SAS Institute, Cary, NC). Evaluation of the fasting lipoproteins at weeks 2, 4, and 6 used one-way ANOVA methods. Analysis of the postprandial 
Table II. Physical Characteristics of Study Subjects

\begin{tabular}{lcccc}
\hline & \multicolumn{4}{c}{ Diet Group } \\
\cline { 2 - 5 } & LC-PUFA & LC-SFA & HC-PUFA & HC-SFA \\
\hline$n$ & 21 & 21 & 20 & 22 \\
Age (yr) & $27.5 \pm 2.9$ & $29.6 \pm 3.1$ & $30.0 \pm 2.9$ & $29.2 \pm 2.8$ \\
*Body Mass Index & $23.4 \pm 1.8$ & $23.8 \pm 2.4$ & $23.7 \pm 1.6$ & $23.5 \pm 2.0$ \\
${ }^{\ddagger}$ Maintenance Energy & & & & \\
$\quad$ Requirement & & & & \\
$\quad$ (kcal) & $3,254 \pm 619$ & $3,188 \pm 412$ & $3,206 \pm 407$ & $3,258 \pm 617$ \\
'BEE (kcal) & $1,757 \pm 124$ & $1,829 \pm 205$ & $1,822 \pm 114$ & $1,761 \pm 169$ \\
"Activity Factor & $1.8 \pm 0.3$ & $1.7 \pm 0.2$ & $1.7 \pm 0.2$ & $1.8 \pm 0.3$ \\
Race (\%) & & & & \\
$\quad$ Caucasian & 67 & 57 & 60 & 64 \\
$\quad$ Asian & 14 & 14 & 20 & 18 \\
Black & 14 & 10 & 10 & 9 \\
Hispanic & 5 & 19 & 10 & 9 \\
\end{tabular}

* Body mass index in weight $(\mathrm{kg}) /$ height $\left(\mathrm{m}^{2}\right) .{ }^{\ddagger}$ Calculated energy intake needed to maintain weight within 3 pounds throughout the study period (BEE $\times$ Activity Factor). ${ }^{8}$ Calculated basal energy expenditure using the Harris-Benedict equation. " Estimated from a modified exercise questionnaire (Maximize Your Body Potential, Joyce D. Nash, Ph.D., Palo Alto: Bull Publishing Company, 1986). Values are means \pm SD.

means obtained over $12 \mathrm{~h}$ required a repeated measures structure for ANOVA. For some analyses, differences from baseline were used to assess the magnitude of change. As an alternative to posthoc tests, we constructed contrast statements rather than test all pairwise multiple comparisons (27). These specific statistical tests were used to evaluate the impact of either the fat and/or cholesterol components of the experimental diets and to subdivide the total statistical variability. Because there are only two levels of each of the two factors (fat and cholesterol) the hypotheses tested by the defined contrasts for the fat and cholesterol components are the same as the two main effects in a two-way factorial design. When a comparison of two diet groups was needed following an ANOVA procedure, a $t$ test on the adjusted group means was carried out. This uses the mean square error as in multiple comparison procedures. Correlations between the fasting values at 2 -wk intervals and between postprandial measurements were assessed using Pearson's correlation coefficient.

\section{Results}

A total of 96 subjects was evaluated and met the eligibility criteria described above. A complete data set was collected from 84 . The remaining individuals withdrew either before ( six subjects) or during (five subjects) the study, or incomplete data were collected (one subject). Mean entry age, body mass index, activity level, and energy requirement of the 84 individuals are shown in Table II. About $60 \%$ of the volunteers in each group was Caucasian, while the remainder was made up of similar proportions of Black, Asian, and Hispanic volunteers (11, 16, and $11 \%$, respectively). This is similar to the proportions of the same groups in the population from which the volunteers were drawn. As assessed by a 5-d food record and 24-h recall, there were no major differences in the nutrients reported consumed by subjects from different ethnic groups, nor between those individuals of all ethnic groups who were later assigned to the four diet groups. The percentage of total calories as fat in the habitual diet reported averaged $35 \%$. The mean calculated cholesterol intake was $350 \mathrm{mg} / \mathrm{d}$.

Fasting cholesterol concentrations. At the beginning of the washout period (day 1) the plasma cholesterol level
Table III. Fasting Plasma Cholesterol and Triglyceride Concentrations

\begin{tabular}{ccrcc}
\hline Diet & Lipid & $\begin{array}{c}\text { Week } 2 \text { end } \\
\text { of washout }\end{array}$ & Week 4 & Week 6 \\
\hline LC-PUFA & TC & $162.1 \pm 25.6$ & $161.8 \pm 26.1$ & $160.3 \pm 24.7$ \\
$(n=21)$ & TG & $75.9 \pm 35.3$ & $79.3 \pm 38.4$ & $72.0 \pm 26.7$ \\
LC-SFA & TC & $163.6 \pm 31.7$ & $166.1 \pm 28.6$ & $165.2 \pm 24.7$ \\
$(n=21)$ & TG & $92.6 \pm 49.1$ & $93.2 \pm 47.2$ & $101.3 \pm 58.8$ \\
HC-PUFA & TC & $166.8 \pm 24.8$ & $185.7 \pm 26.1^{*}$ & $186.0 \pm 27.4^{\ddagger}$ \\
$(n=20)$ & TG & $73.4 \pm 22.7$ & $80.8 \pm 26.0$ & $80.8 \pm 22.0$ \\
HC-SFA & TC & $164.2 \pm 22.0$ & $196.0 \pm 23.9^{*}$ & $193.5 \pm 29.5^{*}$ \\
$(n=22)$ & TG & $65.7 \pm 19.2$ & $85.6 \pm 33.2^{\ddagger}$ & $83.3 \pm 29.2^{\ddagger 8}$ \\
& & & &
\end{tabular}

Values are means $\pm 1 \mathrm{SD}$. Increase from end of washout compared with the change after either LC diet for the same time interval. $* P \leq 0.0001$. ${ }^{\ddagger} P \leq 0.003$. ${ }^{8}$ HC-SFA vs LC-PUFA only.

(mean \pm SD) for all subjects was $172 \pm 28 \mathrm{mg} / \mathrm{dl}^{-1}$ and plasma triglyceride was $86 \pm 37 \mathrm{mg} / \mathrm{dl}$. LDL-C was $112 \pm 23 \mathrm{mg} / \mathrm{dl}$ and HDL-cholesterol was $49 \pm 13 \mathrm{mg} / \mathrm{dl}$. Day 1 plasma total and LDL-C levels were slightly lower for Caucasians than for nonCaucasians (TC: 168 vs $179 \mathrm{mg} / \mathrm{dl}, P=0.15$; LDL-C: 107 vs $119 \mathrm{mg} / \mathrm{dl}, P=0.03$ ). After $14 \mathrm{~d}$ of the washout diet the mean LDL fasting value in Caucasians $(101 \mathrm{mg} / \mathrm{dl}$ on average) was now much lower compared with the other three groups (Asians $121 \mathrm{mg} / \mathrm{dl}$; Blacks $121 \mathrm{mg} / \mathrm{dl}$; Hispanics $118 \mathrm{mg} / \mathrm{dl}$ ) (fourway comparison, $P=0.006$ ). The $P$ values for the difference between LDL values at day 15 in Caucasians compared with Asians, Blacks, and Hispanics were 0.03, 0.01, and 0.02, respectively. As a result when the Caucasian group was compared with the combined non-Caucasians, mean fasting total cholesterol and specifically its LDL component, were significantly higher for non-Caucasians after the washout diet $(P=0.002$ and 0.002 , respectively). Mean fasting IDL cholesterol was also higher after the washout diet in the non-Caucasians (5.4 vs 4.2 $\mathrm{mg} / \mathrm{dl}, P=0.02)$ as was the free cholesterol ( $44.7 \mathrm{vs} 40.4 \mathrm{mg}$ / $\mathrm{dl}, P=0.004)$. At the end of the washout period the mean concentration of VLDL cholesterol in all subjects was 7.6 \pm 5.6 $\mathrm{mg} / \mathrm{dl}$, and that of HDL $43.8 \pm 10.5 \mathrm{mg} / \mathrm{dl}$. The concentration of triglyceride in VLDL was $41.0 \pm 29.0 \mathrm{mg} / \mathrm{dl}$ and in IDL it was $4.0 \pm 4.7 \mathrm{mg} / \mathrm{dl}$. There was no significant difference in these concentrations when Caucasian and non-Caucasian groups were compared.

Mean cholesterol concentrations among the four diet groups were found to differ after the first $2 \mathrm{wk}$ of the experimental diets (Table III). Those continuing on the LC-PUFA diet (both Caucasians and non-Caucasians) did not demonstrate any significant change in mean values over the experimental period in either plasma or LDL cholesterol. This result indicates that equilibrium had been reached during the 2-wk washout period. In the LC-SFA group, whose diet now contained more saturated and less polyunsaturated fat but a similar low cholesterol content, there was also little mean change in fasting plasma cholesterol level. At the beginning of the experimental period, mean plasma cholesterol was $164 \mathrm{mg} / \mathrm{dl}$, while after 2 and 4 wk of saturated fat diet it averaged 166 and $165 \mathrm{mg} / \mathrm{dl}$, respectively (Table III). The change in saturated fat content (LC-PUFA vs LC-SFA) did not affect mean LDL-C overall or within the non-Caucasian subgroup, but when the Caucasian group was 
Table IV. Mean Weeks 2 and 4 Fasting Plasma LDL-C by Diet and Ethnic Group

\begin{tabular}{|c|c|c|c|c|c|c|}
\hline \multirow[b]{2}{*}{ Diet } & \multicolumn{2}{|c|}{$\begin{array}{c}\text { Total } \\
(n=84)\end{array}$} & \multicolumn{2}{|c|}{$\begin{array}{c}\text { Caucasian } \\
(n=52)\end{array}$} & \multicolumn{2}{|c|}{$\begin{array}{l}\text { Non-Caucasian } \\
\quad(n=32)\end{array}$} \\
\hline & Mean & Change & Mean & Change & Mean & Change \\
\hline \multirow[t]{2}{*}{ LC-PUFA } & 106.2 & & 103.8 & & 111.0 & \\
\hline & 104.2 & -2.0 & 101.7 & -2.1 & 109.2 & -1.8 \\
\hline \multirow[t]{2}{*}{ LC-SFA } & 108.7 & & 96.3 & & 123.9 & \\
\hline & 112.0 & 3.3 & 102.6 & 6.3 & 123.2 & -0.7 \\
\hline \multirow[t]{2}{*}{ HC-PUFA } & 109.2 & & 100.6 & & 122.1 & \\
\hline & 124.8 & 15.6 & 119.0 & 18.4 & 133.5 & 11.4 \\
\hline \multirow[t]{2}{*}{ HC-SFA } & 108.7 & & 102.0 & & 120.4 & \\
\hline & 134.1 & 25.4 & 132.9 & 30.9 & 136.2 & 15.8 \\
\hline
\end{tabular}

Comparison of fasting LDL-C by diet at week 2: Total, $P=0.98$; Caucasian, $P=0.74$; non-Caucasian, $P=0.84$. LDL-C values are from the end of the washout (week 2 of study) and after 2 wk of experimental diet (week 4 of study).

\begin{tabular}{|c|c|c|c|}
\hline Change in fasting LDL-C: & Total & Caucasians & Non-Caucasians \\
\hline \multicolumn{4}{|l|}{ Cholesterol effect } \\
\hline LC-PUFA vs HC-PUFA & $P<0.0001$ & $<0.0001$ & NS \\
\hline LC-SFA vs HC-SFA & $P<0.0001$ & $<0.0001$ & 0.04 \\
\hline \multicolumn{4}{|l|}{ Fat effect } \\
\hline LC-PUFA vs LC-SFA & $P=\mathrm{NS}$ & 0.05 & NS \\
\hline HC-PUFA vs HC-SFA & $P=0.02$ & 0.004 & NS \\
\hline
\end{tabular}

considered separately, LDL-C means differed between the end of the washout period and after 2 wk of the experimental diet $(P=0.05$, Table IV). A different result was obtained with the HC-SFA diet, which compared to the washout had higher levels of both saturated fat and cholesterol. In this case there was an overall mean increase of $32 \mathrm{mg} / \mathrm{dl}$ in plasma total cholesterol after 2 wk. Most of the change $(+25 \mathrm{mg} / \mathrm{dl}$ on average) was in LDL (Fig. 2). There was a significantly greater response in Caucasian than in non-Caucasian subjects (Table IV) (30.9 $\mathrm{mg} / \mathrm{dl}$ vs $15.8 \mathrm{mg} / \mathrm{dl}, P=0.004)$. On the HC-PUFA diet, where increased cholesterol was combined with more polyunsaturated fat, total plasma cholesterol also increased compared to the washout period. The mean increment was $+19 \mathrm{mg} / \mathrm{dl}$ in

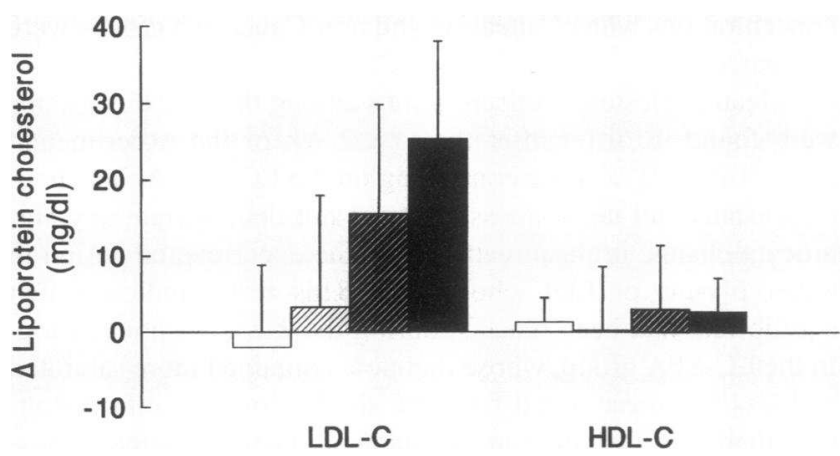

Figure 2. Changes in the concentration of LDL and HDL cholesterol on the four test diets during the experimental period. The data shown are the difference in the mean fasting values between the end of the washout period and after $2 \mathrm{wk}$ of each experimental diet. (Open bars) LC-PUFA; (light shading) LC-SFA; (heavy shading) HC-PUFA; (filled bars), HC-SFA. this case ( $P<0.001$ compared to washout), significantly less than with the HC-SFA diet $(P=0.001)$. This same relationship was observed in the LDL-C component $(P=0.02$, Table IV). However the response of the non-Caucasians to the HC-SFA and the HC-PUFA diets did not differ significantly. These data indicate that in the presence of either high or low cholesterol intake, there was a significant response to saturated fat among the Caucasian subjects but not among the non-Caucasians.

The distributions of individual changes in LDL-C over the first 2 wk of the experimental diets for the LC-PUFA, LC-SFA, HC-PUFA, and HC-SFA diets are shown in Fig. 3. In the case of the HC-SFA diet group, it was observed that seven of eight values for the non-Caucasian subjects were less than the mean for the Caucasians.

There was no significant correlation between LDL cholesterol concentration at the end of the washout period and any subsequent increase in LDL cholesterol found on the HC-PUFA or HC-SFA diets $(r=0.27, P=0.09)$. On all of the diets, there was no further significant change in plasma or LDL cholesterol levels when the same diet was continued for a further 2 wk.

After the washout there was no difference in mean fasting HDL cholesterol when analyzed by diet or race. The magnitude of change from the washout during the experimental diet period (Fig. 2) did not differ statistically by diet either overall or for any pairwise combination.

Postprandial effects on plasma cholesterol levels. There was significant difference among the fasting mean plasma free cholesterol concentrations at weeks four and six in that on average, higher levels were observed in individuals consuming cholesterol-rich diets (four-way ANOVA, $P=0.005$ and 0.006 , respectively); but acute postprandial changes were similar with all of the four test meal cholesterol/fat combinations. The re- 


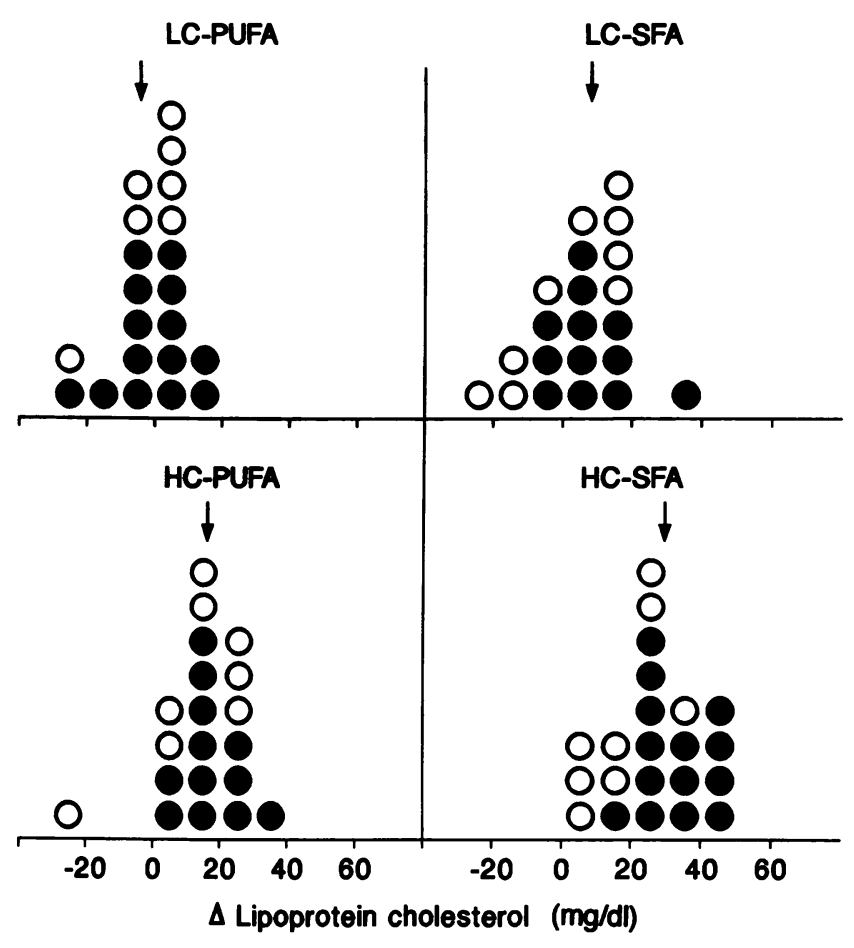

Figure 3. LDL cholesterol response of individual donors in response to the four experimental diets. Values shown are the difference between fasting concentrations at the end of the washout period and after two weeks of experimental diet. (Closed circles) Caucasian subjects, (open circles) non-Caucasian subjects. Arrows indicate mean LDL-C for the combined diet group.

sults after an HC-SFA test meal at weeks 2, 4, and 6 are shown in Table V. Values rose by $1-2 \mathrm{mg} / \mathrm{dl}$ at $3-9 \mathrm{~h}$ and then decreased again to fasting levels at $12 \mathrm{~h}$.

The pattern of EC response above fasting levels was also similar with each of the four test meals at 2, 4, and $6 \mathrm{wk}$. HDL EC levels increased from fasting levels by $12 \mathrm{~h}$ postprandially $(+1.6,1.8$, and $1.9 \mathrm{mg} / \mathrm{dl}$ on average at weeks 2,4 , and 6$)$. This was a significant difference at each time point $(P$ $<0.0001)$. Fig. 4 displays the magnitude of change from fasting for the HC-SFA diet at weeks 2, 4, and 6. The rest of the postprandial increase in cholesteryl ester was found in LDL.

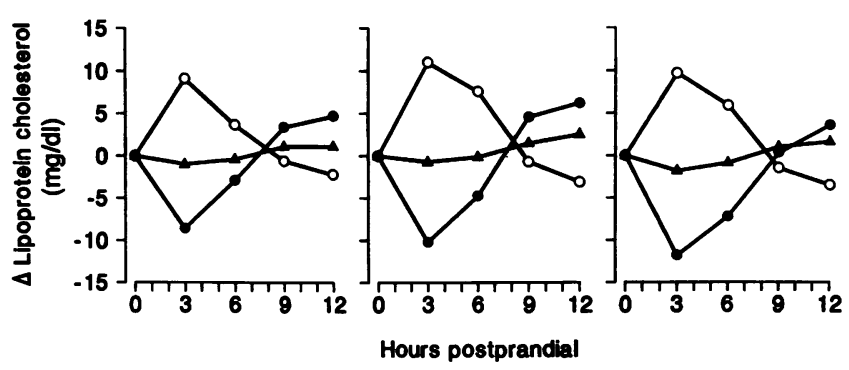

Figure 4. Changes from fasting in lipoprotein cholesterol content in postprandial plasma at the end of the 2-wk washout period (left) and after 2 wk (center) and 4 wk (right) consumption of the HC-SFA diet. Values are the mean differences of all subjects in this experimental group. (Open circles) VLDL cholesterol; (closed circles) IDL + LDL cholesterol; (closed triangles) HDL cholesterol. Comparable changes were found in the other diet groups.

These changes were part of a marked redistribution of cholesterol between plasma lipoprotein classes. There was first a significant but transient postprandial increase of VLDL cholesterol $(P<0.0001)$ peaking simultaneously with triglyceride after 3 $h$. There was a simultaneous but opposite pattern in the cholesterol content of the LDL+ IDL fraction. Later in the postprandial cycle (9-12 h) these effects were reversed, so that the major final effect $(12 \mathrm{~h})$ was an increase in LDL+ IDL EC over initial levels $(P<0.0001)$.

Fasting triglyceride levels. After the washout, mean fasting triglyceride values differed somewhat among the four diet groups, with those individuals selected to receive the LC-SFA diet having a higher concentration (Table III, $P=0.07$ ). Therefore, subsequent changes in TG concentration were related to baseline values obtained at the end of the washout period. After $2 \mathrm{wk}$ of the experimental diets a significant increase in mean TG occurred among those receiving a high cholesterol diet $(+13.9 \mathrm{mg} / \mathrm{dl}$ for the combined HC-PUFA and HC-SFA groups vs $+2.0 \mathrm{mg} / \mathrm{dl}$ for the combined LC-PUFA and LCSFA groups, $P=0.003$ ). This difference may, however, reflect at least in part the higher initial concentration of TG in the LCSFA group. No further change occurred between weeks 4 and 6. In addition, no difference between Caucasians and non-Caucasians occurred in fasting TG after the washout phase or in the increase in TG at weeks 4 and 6 of the study.

Table V. Plasma Fasting and Postprandial Free and Esterified Cholesterol after the HC-SFA Diet

\begin{tabular}{|c|c|c|c|c|c|}
\hline \multirow[b]{2}{*}{ Study week } & \multicolumn{5}{|c|}{ Hours postprandial } \\
\hline & 0 & 3 & 6 & 9 & 12 \\
\hline \multicolumn{6}{|l|}{ Week 2} \\
\hline $\mathrm{FC}$ & $41.4 \pm 6.5$ & $42.7 \pm 6.3$ & $42.2 \pm 6.1$ & $42.7 \pm 6.5$ & $41.8 \pm 6.0$ \\
\hline $\mathrm{EC}$ & $122.9 \pm 15.9$ & $121.0 \pm 15.1$ & $122.4 \pm 13.3$ & $125.5 \pm 14.0$ & $126.0 \pm 13.8$ \\
\hline \multicolumn{6}{|l|}{ Week 4} \\
\hline FC & $50.3 \pm 8.4$ & $51.6 \pm 8.0$ & $52.1 \pm 8.5$ & $51.6 \pm 8.5$ & $50.7 \pm 8.9$ \\
\hline EC & $145.7 \pm 17.0$ & $144.5 \pm 16.6$ & $146.8 \pm 18.0$ & $149.7 \pm 18.6$ & $150.8 \pm 19.2$ \\
\hline \multicolumn{6}{|l|}{ Week 6} \\
\hline FC & $51.0 \pm 8.2$ & $51.8 \pm 9.1$ & $52.3 \pm 8.8$ & $51.6 \pm 8.1$ & $51.1 \pm 9.1$ \\
\hline EC & $142.5 \pm 21.6$ & $137.8 \pm 21.9$ & $139.1 \pm 21.7$ & $141.7 \pm 21.1$ & $144.0 \pm 23.6$ \\
\hline
\end{tabular}

Values shown are mean \pm SD. 


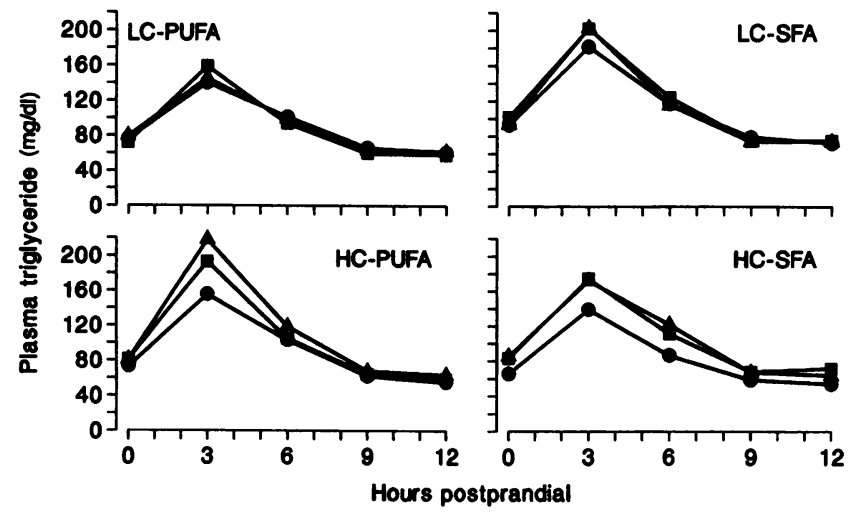

Figure 5. Changes in plasma triglyceride during postprandial lipemia for each diet. (Circles) end of the 2-wk washout diet period; (triangles) after $2 \mathrm{wk}$ of experimental diets; (squares), after $4 \mathrm{wk}$ of the experimental diets. Values shown are means for all subjects in each experimental group.

Postprandial triglyceride concentrations. The absorption of dietary fat was followed by a doubling of the fasting level of plasma triglyceride $3 \mathrm{~h}$ postprandially, typically followed by a decline below fasting levels at 9-12 h (Fig. 5). Fasting triglyceride was highly predictive of the magnitude of postprandial response at $3 \mathrm{~h}$ at weeks 2,4 , and $6(r=0.70, P<0.001)$. We also found a lower $3-\mathrm{h}$ postprandial triglyceride concentration in Caucasians compared with non-Caucasians (at the end of the washout period, $137.6 \mathrm{vs} 180.0 \mathrm{mg} / \mathrm{dl}, P=0.03$; after 2 wk of experimental diet, $169.2 \mathrm{vs} 208.0 \mathrm{mg} / \mathrm{dl}, P=0.04$; after $4 \mathrm{wk}$ of experimental diet, $167.8 \mathrm{vs} 200.0 \mathrm{mg} / \mathrm{dl}, P=0.06$ ). For the entire postprandial interval these differences persisted.

Fasting and postprandial plasma LCAT activity. There was a significant difference in mean LCAT activity among the four diet groups at the end of the washout period, with the LC-PUFA and LC-SFA groups (20.0 and $21.3 \mu \mathrm{g} / \mathrm{ml}$ per $\mathrm{h}$, respectively) being higher than the HC-PUFA and HC-SFA groups (15.5 and $14.9 \mu \mathrm{g} / \mathrm{ml}$ per $\mathrm{h}$, respectively) $(P=0.0004)$. After $2 \mathrm{wk}$ of the experimental diets the mean fasting rate of LCAT activity decreased for those consuming a low cholesterol diet, while it increased with a high cholesterol diet, resulting in equivalent mean fasting rates (LC-PUFA 16.7, LC-SFA 18.0, HC-PUFA 17.7, and HC-SFA $17.3 \mu \mathrm{g} / \mathrm{ml}$ per $\mathrm{h}$, respectively). After a further $2 \mathrm{wk}$ ( $4 \mathrm{wk}$ in all on the experimental diets) mean fasting values in each case tended to return towards their original values (LC-PUFA 18.0, LC-SFA 19.7, HC-PUFA 16.2, and HC-SFA $16.6 \mu \mathrm{g} / \mathrm{ml}$ per $\mathrm{h}$, respectively $)(P=0.18)$.

All four diets resulted in a generally similar pattern in the 12-h postprandial period after 2 wk of experimental diet (Fig. 6 ) but with a significant difference among the five means obtained at 3 -h intervals $(P<0.0001)$. Activities increased postprandially above fasting baseline values with a peak at $6 \mathrm{~h}$, lagging from the maximum postprandial triglyceride concentration $(3 \mathrm{~h})$, and then decreased to close to their original values by $12 \mathrm{~h}$, indicating a curvilinear effect. At week 4 the highest postprandial response was found in the HC-PUFA group, while a lower response was found with the HC-SFA diet. However, the difference in the level of postprandial response due to diet (Fig. 6) was of only borderline significance $(P=0.06)$. No difference occurred at $6 \mathrm{wk}$.

There was a modest but significant difference between Cau-

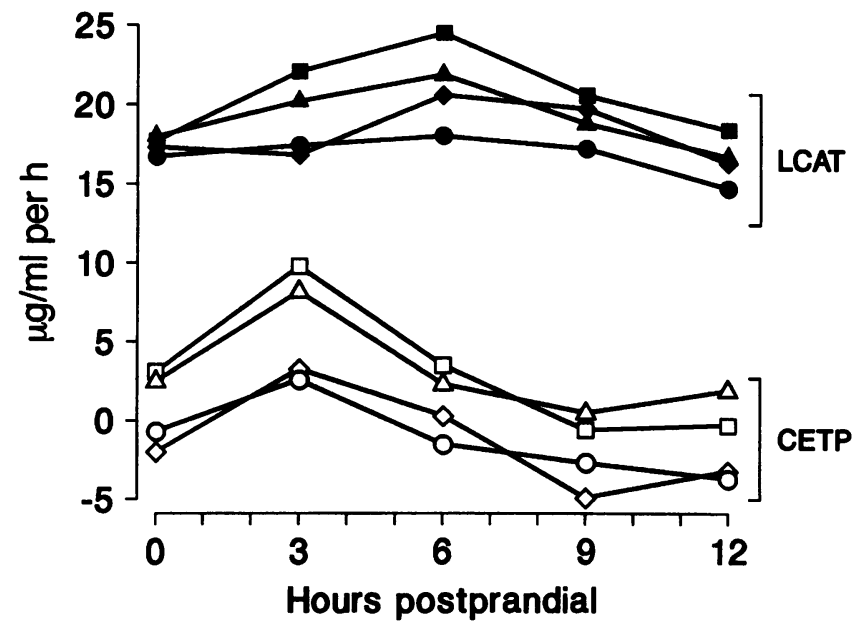

Figure 6. Fasting and postprandial values for LCAT activity (above) and CETP activity (below) after 2 wks on the four experimental diets. (Circles) LC-PUFA; (triangles) LC-SFA; (squares) HC-PUFA; (diamonds) HC-SFA.

casian and non-Caucasian groups in baseline fasting LCAT activity $(16.7 \pm 5.7$ vs $19.8 \pm 6.6 \mu \mathrm{g} / \mathrm{ml}$ per $\mathrm{h}, P=0.03)$, along with a difference in the test meal response over $12 \mathrm{~h}$ of LCAT values (wk 2) $(P=0.05)$. This ethnic difference was not seen after 4 wk of experimental diet, however.

A significant correlation was found between LCAT and triglyceride values both in fasting plasma and $3 \mathrm{~h}$ postprandially. In fasting plasma, this correlation at weeks 2,4 , and 6 was $r$ $=0.56, P<0.001 ; r=0.32, P=0.003$; and $r=0.41, P$ $<0.001$. In $3 \mathrm{~h}$ postprandial plasma, the correlation at weeks 2,4 , and 6 was $r=0.51, P<0.001 ; r=0.36, P=0.001$; and $r=0.39, P<0.001$.

Fasting and postprandial plasma CETP activities. On average there was little or no net transfer of cholesteryl esters from HDL to VLDL and LDL in fasting plasma after the washout period (overall mean $-0.5 \mu \mathrm{g} \mathrm{ml} / \mathrm{h}$ EC transferred, vs an average of $18.0 \mu \mathrm{g} / \mathrm{ml}$ per h EC synthesized by LCAT). After 2 wk of the experimental diets there was a significant difference among the $0-12-\mathrm{h}$ interval means $(P<0.0001)$ with maximal activity with all of the four diets at $3 \mathrm{~h}$. coincident with the maximum of plasma triglyceride (Fig. 6). A significant diet effect was also observed at this time $(P=0.006)$ with the highest activities found with the HC-PUFA and HC-SFA groups. However, the diet and postprandial effects became nonsignificant after 4 wk of the experimental diets. Lower CETP values $(P=0.03)$ were found in the Caucasians after $2 \mathrm{wk}$ of the study diets, but were not observed after $4 \mathrm{wk}$.

\section{Discussion}

In this study, we measured the independent contributions of changes in the proportions of saturated and polyunsaturated fat and of cholesterol to LDL-C changes in Caucasian and nonCaucasian young men. The data of Hegsted and Keys and subsequent work (3-5) predicted that with the diet changes used, these modifications in fat and cholesterol would each contribute substantially to increases in total cholesterol, and that these would be additive (Table VI).

In agreement with earlier reports $(28,29)$ we observed con- 
Table VI. Changes in Plasma Cholesterol Predicted and Observed

\begin{tabular}{lcccc}
\hline & LC-PUFA & LC-SFA & HC-PUFA & HC-SFA \\
\hline & $m g / d l$ & & & \\
Keys equation & 0 & +18.5 & +13.1 & +33.7 \\
Hegsted equation & 0 & +16.5 & +15.4 & +35.7 \\
Observed (all) & -0.3 & +2.4 & +18.8 & +31.8 \\
Observed (Caucasian) & +0.5 & +4.6 & +17.6 & +35.7 \\
Observed (non-Caucasian) & -1.8 & -0.4 & +20.6 & +24.9 \\
& & & & \\
\hline Analysis of variance: & \multirow{2}{*}{ Total } & Caucasian & Non-Caucasian \\
\hline Cholesterol effect & & & & \\
$\quad$ LC-PUFA vs HC-PUFA & $P<0.0001$ & $P=0.0002$ & $P=0.007$ \\
$\quad$ LC-SFA vs HC-SFA & $P<0.0001$ & $P<0.0001$ & $P=0.002$ \\
Fat saturation effect & & & & \\
$\quad$ LC-PUFA vs LC-SFA & $P=0.48$ & $P=0.34$ & $P=0.85$ \\
HC-PUFA vs HC-SFA & $P=0.001$ & $P=0.0001$ & $P=0.57$ \\
& & & & \\
\hline
\end{tabular}

siderable variation in the response to dietary fat and cholesterol. However the experimental data showed unequivocally that in our population, increasing saturated fat at the expense of polyunsaturated fat alone had little effect on average total cholesterol and LDL-C concentrations.

In hamsters and at least some nonhuman primates, the hypercholesterolemic effect of dietary saturated fat is greatly amplified by addition of cholesterol to the diet (30), but this effect has not been observed in guinea pigs (31). It is generally believed that the effect of dietary saturated fats in humans is independent of dietary cholesterol. However, from a recent analysis of literature data, Hayes and Khosla have concluded that in normolipidemic subjects consuming $<300 \mathrm{mg}$ cholesterol daily, the major dietary saturated fatty acid, palmitic acid, has no effect upon plasma cholesterol concentration, most of the observed variance being explained by intake of myristic and linoleic acids (2). At higher levels of dietary cholesterol, palmitic acid may be hypercholesterolemic, an effect attributed to partial downregulation of the LDL receptor by dietary cholesterol (2). In our subjects ingesting $\sim 200 \mathrm{mg}$ cholesterol daily, little effect on total cholesterol or LDL-C was observed when the polyunsaturated/saturated fat ratio was reduced from 0.83 to 0.38 , achieved by increases in consumption of myristic and stearic as well as palmitic acid.

The converse situation-reduced effect of dietary cholesterol on serum cholesterol and LDL-C when polyunsaturaterich diets are fed-has been observed by some, but not all investigators, as reviewed elsewhere $(3,32,33)$. In a systematic study of this question, Schonfeld et al. varied the $\mathrm{P} / \mathrm{S}$ ratio of the diet from 0.25 to $0.4,0.8$, and 2.5 in normolipemic young men fed diets containing $300 \mathrm{mg}$ cholesterol or with the addition of three or six eggs (calculated as containing an additional 750 or 1,500 mg cholesterol) (7). They found graded responses to a given amount of added cholesterol. At the highest $\mathrm{P} / \mathrm{S}$ ratio, no effect of adding 750 or $1,500 \mathrm{mg}$ of cholesterol on total cholesterol or LDL-C was observed in six subjects. In our study, the result of adding $\sim 400 \mathrm{mg}$ cholesterol to the diet at a P/S ratio of $\sim 0.8$ was slightly greater than that predicted by the Keys and Hegsted equations, and the combined effect of a cho- lesterol and saturated fatty acid-rich diet was close to that predicted by these equations.

One reason for the differences found in the current study may be the inclusion of a substantial proportion of non-Caucasians in the study population. On average these subjects showed little or no response to replacement of saturated for polyunsaturated fat (alone or in combination with cholesterol) although their response to cholesterol was similar to that of Caucasians (Table VI). Ethnicity was not given in most previous studies, although the number of non-Caucasians was unlikely to have been large. Even in Caucasians the effect of replacing saturated for polyunsaturated fat was much less than predicted when dietary cholesterol was low. It is possible that the relatively high level of monounsaturates in this study ( $\sim 40 \%$ of total fat calories) could have inhibited the hypercholesterolemic effect of fat saturation; but this seems unlikely because response to the HCSFA diet was similar to that predicted by the Keys and Hegsted equations. It seems more likely that saturated fat did interact with cholesterol in this study to increase cholesterol's hypercholesterolemic effect, at least in some individuals. If this interpretation is correct this interaction may represent a factor, possibly genetic, that is relatively common in Caucasians, but much less common in non-Caucasians.

In contrast to these findings with plasma cholesterol, fasting and postprandial triglyceride levels did not differ among ethnic groups. Plasma fasting triglyceride was unaffected by dietary fat saturation. This probably reflects the stable body weight of the subjects, a major determinant of plasma triglyceride levels (34). Postprandial triglyceride response was highly correlated with fasting triglyceride concentration, consistent with previous data (35), but was unaffected by the basal diet.

Both LCAT and CETP activities increased postprandially, consistent with previous reports $(13,14,36)$. CETP increased with triglyceride, peaking at $\sim 3 \mathrm{~h}$, probably because VLDL triglyceride was rate limiting for cholesteryl ester transfer (37). The maximal increase in LCAT occurred later $(6-9 \mathrm{~h})$. Fasting LCAT rates were not affected by diet, and the general shape of the postprandial response curve of LCAT was similar regardless of the composition of the test meal consumed. In the case of CETP, fasting rates were increased after 2 wk consumption of the HC-PUFA and LC-SFA diets. The mean increase in CETP activity found with the HC-PUFA and LC-SFA diets $(\sim+5$ $\mu \mathrm{g} / \mathrm{ml}$ per $\mathrm{h}$ averaged over the $12-\mathrm{h}$ period of measurement) would represent an increase of $(5 \times 60 \times 100)$ or $+30 \mathrm{mg} /$ $\mathrm{dl}$ in VLDL-C and LDL-C over the estimated $60-\mathrm{h}$ residence time of LDL (38). However, there was no parallel change in LDL-C. In LC-SFA group, LDL-C was almost unchanged; in the HC-SFA group, LDL-C increased but CETP activity was unchanged by diet; while in the HC-PUFA group, CETP activity was increased. Over a longer period ( 4 wk) while LDL-C was maintained, CETP activity decreased once more toward baseline in the LC-SFA and HC-PUFA groups. These results indicate that factors other than CETP activity are preeminent in determining LDL-C. In many mammals, probably including humans, the effect of saturated fat is exerted mainly at the level of the LDL receptor (30). This may be the longer-term, CETPindependent mechanism that maintains elevated LDL-C when mean CETP activity, initially increased, has returned to its original levels.

Findings in this study, including the influence of dietary cholesterol as related to dietary fat saturation, differences between ethnic groups, and trends in LCAT and CETP activity, 
will all need to be confirmed by further research to determine if they apply more broadly to the population. Our subjects were relatively young. It is possible that with age the importance of dietary fat saturation (relative to cholesterol) increases. There may also be unrecognized differences in physiology, perhaps reflecting lifestyle factors such as exercise, body mass index, or habitual diet that distinguish this population sample, although Caucasians and non-Caucasians appeared not to differ in these respects. If the differing dietary responses of Caucasians and other ethnic groups observed here apply to the greater population, this could have important implications for public health and education.

\section{Acknowledgments}

We gratefully acknowledge the expert assistance provided by M. Cansanay, D. Devereux, J. Diehl, A. Hariri, and D. Patra; and by the clinical nursing staff of the General Clinical Research Center. We thank M. Spitalny, Information Technology Services, University of California at San Francisco, for developing the custom software used in this study.

Funding for this research was provided by the National Institutes of Health grant HL-41224 and Arteriosclerosis SCOR HL-14237. It was also funded by the National Dairy Promotion and Research Board by an award administered in cooperation with the National Dairy Council. General Clinical Research Center support was funded through the National Institutes of Health grant RR-00079.

\section{REFERENCES}

1. Grundy, S. M., and M. A. Denke. 1990. Dietary influences on serum lipids and lipoproteins. J. Lipid Res. 31:1149-1172.

2. Hayes, K. C., and P. Khosla. 1992. Dietary fatty acid thresholds and cholesterolemia. FASEB (Fed. Am. Soc. Exp. Biol.) J. 6:2600-2607.

3. Hegsted, D. M., L. M. Aussman, J. A. Johnson, and G. E. Dallal. 1993 Dietary fat and serum lipids: an evaluation of the experimental data. Am. J. Clin. Nutr. 57:875-883.

4. Keys, A., J. T. Anderson, and F. Grande. 1965. Serum cholesterol response to changes in diet. The effect of cholesterol in the diet. Metab. Clin. Exp. 14:759765 .

5. Hegsted, D. M., R. B. McGandy, M. L. Myers, and F. J. Stare. 1965. Quantitative effects of dietary fat on serum cholesterol in man. Am. J. Clin. Nutr 17:281-295.

6. National Heart-Diet Research Group. 1968. National heart-diet study final report. Circulation. 37(Suppl. 1):260-274

7. Schonfeld, G., W. Patsch, L. L. Rudel, C. Nelson, M. Epstein, and R. E. Olson. 1982. Effects of dietary cholesterol and fatty acids on plasma lipoproteins J. Clin. Invest. 69:1072-1080.

8. Kestin, M., P. M. Clifton, I. L. Rouse, and P. J. Nestel. 1989. Effect of dietary cholesterol in normolipemic subjects is not modified by the nature and amount of dietary fat. Am. J. Clin. Nutr. 50:528-532.

9. Derr, J., P. M. Kris-Etherton, T. A. Pearson, and F. H. Seligson. 1993. The role of fatty acid saturation on plasma lipids, lipoproteins and apolipoproteins The plasma total and low-density lipoprotein cholesterol response to individual fatty acids. Metab. Clin. Exp. 42:130-134.

10. Ng, T. K. W., K. C. Hayes, G. F. DeWitt, M. Jegathesan, N. Satgunasingam, A. S. H. Ong, and D. Tan. 1992. Dietary palmitic and oleic acids exert simila effects on serum cholesterol and lipoprotein profiles in normocholesterolemic men and women. J. Am. Coll. Nutr. 11:383-390.

11. Fielding, P. E., and C. J. Fielding. 1992. Dynamics of lipoprotein transport in the circulatory system. In Biochemistry of Lipids and Membranes. J. E. Vance and D. E. Vance, editors. Elsevier Science Publishing Co., New York. 427-459.

12. Fielding, P. E., Y. Ishikawa, and C. J. Fielding. 1989. Apolipoprotein E mediates binding of normal very low density lipoprotein to heparin but is not required for high affinity receptor binding. J. Biol. Chem. 264:12462-12466.

13. Castro, G. R., and C. J. Fielding. 1985. Effects of postprandial lipemia on plasma cholesterol metabolism. J. Clin. Invest. 75:874-882.

14. Tall, A., D. Sammett, and E. Granot. 1986. Mechanisms of enhanced cholesteryl ester transfer from high density lipoproteins to apoliprotein B-containing lipoproteins during alimentary lipemia. J. Clin. Invest. 77:1163-1172.

15. Abbey, M., P. Clifton, M. Kestin, B. Belling, and P. Nestel. 1990. Effect of fish oil on lipoproteins, lecithin: cholesterol acyltransferase and lipid transfer protein activity in humans. Arteriosclerosis. 10:85-94.

16. Quinet, E., A. Tall, R. Ramakrishnan, and L. Rudel. 1991. Plasma lipid transfer protein as a determinant of the atherogenicity of monkey plasma lipoproteins. J. Clin. Invest. 87:1559-1566.

17. Applebaum-Bowden, D., S. M. Haffner, E. Hartsook, K. H. Luk, J. J. Albers, and W. R. Hazzard. 1984. Downregulation of the low density lipoprotein receptor by dietary cholesterol. Am. J. Clin. Nutr. 39:360-367.

18. Packard, C. J., L. McKinney, K. Carr, and J. Shepherd. 1983. Cholesterol feeding increases low density lipoprotein synthesis. J. Clin. Invest. 72:45-51.

19. Lipid Research Clinics Population Studies Data Book. 1980. Vol. 1. Publication No. 80-1527, DHHS (NIH), Washington, DC, 136 pp.

20. Harris, J. A., and F. G. Benedict. 1919. A Biometric Study of basa metabolism in Man. Publication No. 279, Carnegie Institution, Washington, DC

21. Allain, C. C., L. S. Poon, C. S. G. Chan, W. Richmond, and P. C. Fu 1974. Enzymatic determination of total serum cholesterol. Clin. Chem. 20:470475 .

22. Bucolo, G., and H. David. 1973. Quantitative determination of serum triglycerides by the use of enzymes. Clin. Chem. 19:476-482.

23. Kostner, G. M. 1976. Enzymatic determination of cholesterol in high density lipoprotein fractions prepared by polyanion precipitation. Clin. Chem 22:695

24. Havel, R. J., H. A. Eder, and J. H. Bragdon. 1955. The distribution and chemical composition of ultracentrifugally separated lipoproteins from human serum. J. Clin. Invest. 34:1345-1353.

25. Fielding, C. J. 1984. Lecithin: cholesterol acyltrans-ferase and cholesterol transport. Methods Enzymol. 111:267-274.

26. Ogawa, Y., and C. J. Fielding. 1984. Assay of cholesteryl ester transfer activity and purification of a cholesteryl ester transfer protein. Methods Enzymol. 111:274-285

27. Snedecor, G. W., and W. G. Cochran. 1967. Statistical Methods. Iowa State University Press, Ames, IA. 6th edition, 346-358.

28. Mistry, P., N. E. Miller, M. Laker, W. R. Hazzard, and B. Lewis. 1981 Individual variation in the effects of dietary cholesterol on plasma lipoproteins and cellular cholesterol homeostasis in man. J. Clin. Invest. 67:493-502.

29. Katan, M. B., A. C. Beynen, J. H. DeVries, and A. Nobels. 1986. Existence of consistent hypo- and hyper-responders to dietary cholesterol in man. Am. J. Epidemol. 125:387-399.

30. Spady, D. K., L. A. Woollet, and J. M. Dietschy. 1993. Regulation of plasma LDL-cholesterol levels by dietary cholesterol and fatty acids. Annu. Rev. Nutr. 13:355-381.

31. Lin, E. C. K., M. L. Fernandez, M. A. Tosca, and D. J. McNamara. 1994 Regulation of hepatic LDL metabolism in the guinea pig by dietary fat and cholesterol. J. Lipid Res. 35:446-457.

32. McNamara, D. J. 1990. Relationship between blood and dietary cholesterol. Adv. Meat Res. 6:63-87.

33. Hopkins, P. N. 1992. Effects of dietary cholesterol on serum cholesterol: a meta-analysis and review. Am. J. Clin. Nutr. 55:1060-1070.

34. Phillips, N. R., R. J. Havel, and J. P. Kane. 1981. Levels and interrelationships of serum and lipoprotein cholesterol and triglycerides. Arteriosclerosis. $1: 13-24$.

35 Cohen, J. C. 1989. Chylomicron triglyceride clearance: comparison of three assessment methods. Am. J. Clin. Nutr. 49:306-313.

36. Marcel, Y. L., and C. Vezina. 1973. Lecithin:cholesterol acyltransferase of human plasma. Role of chylomicrons, very low, and low density lipoproteins in the reaction. J. Biol. Chem. 248:8254-8259.

37. Mann, C. J., F. T. Yen, A. M. Grant, and B. E. Bihain. 1991. Mechanism of plasma cholesteryl ester transfer in hypertriglyceridemia. J. Clin. Invest. 88:20592066.

38. Kesaniemi, Y. A., W. F. Beltz, and S. M. Grundy. 1985. Comparisons of metabolism of apolipoprotein B in normal subjects, obese patients and patients with coronary heart disease. J. Clin. Invest. 76:586-595. 